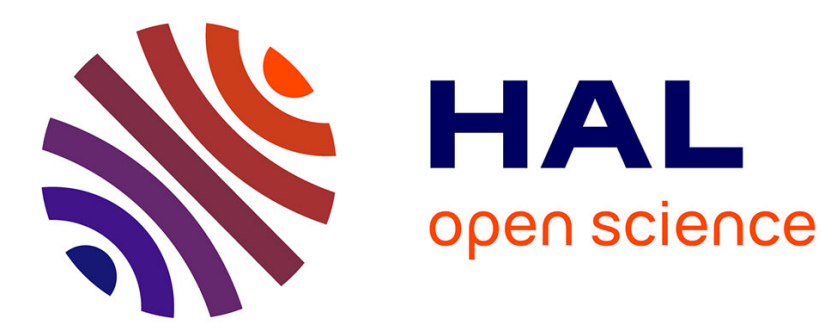

\title{
The G0 backangle measurements
}

S. Kox

\section{To cite this version:}

S. Kox. The G0 backangle measurements. International Workshop: From Parity Violation to Hadronic Structure and more.. Part 3 - PAVI06, May 2006, Milos Island, Greece. pp.469-472, 10.1140/epja/i2006-10420-2 . in2p3-00092546

\section{HAL Id: in2p3-00092546 https://hal.in2p3.fr/in2p3-00092546}

Submitted on 11 Sep 2006

HAL is a multi-disciplinary open access archive for the deposit and dissemination of scientific research documents, whether they are published or not. The documents may come from teaching and research institutions in France or abroad, or from public or private research centers.
L'archive ouverte pluridisciplinaire HAL, est destinée au dépôt et à la diffusion de documents scientifiques de niveau recherche, publiés ou non, émanant des établissements d'enseignement et de recherche français ou étrangers, des laboratoires publics ou privés. 


\title{
The $G^{0}$ Backangle Measurements
}

\author{
Serge Kox (for the $G^{0}$ Collaboration $^{\text {a }}$ ) \\ LPSC, IN2P3-UJF, 53 Avenue des Martyrs, 38026 Grenoble Cedex, France \\ Received: date / Revised version: date
}

\begin{abstract}
This contribution presents the status, at the time of this conference, of the second phase of the $G^{0}$ experiment. This experiment is being performed in Hall $\mathrm{C}$ of Jefferson Laboratory. It measures PV asymmetries in elastic electron-proton and quasi-elastic electron-deuteron scattering. Details of the experimental setup are given, together with the first data collected in 2006 at backward angles.
\end{abstract}

PACS. 13.60.Fz Elastic and Compton Scattering - 13.40.Gp Electromagnetic Form Factors - 14.20.Dh Protons and Neutrons

\section{Introduction}

A good understanding of the role of its sea of gluons and pairs of quarks and antiquarks is crucial to our knowledge of the structure of the nucleon. This is, in particular, the case for the search for possible contributions of strange quark-antiquarks pairs to nucleon properties. To this end, parity-violation (PV) experiments in electronnucleon scattering have become, over the years, a reliable and powerful technique for extracting information on the nucleon internal structure [1]. These experiments have already published a set of results $[2-5]$, but a new round of measurements is underway, or planned, to allow for the separation of the neutral weak form factors and the effective axial current of the nucleon. Combined with the known electromagnetic form factors, these new data permit in particular the isolation of the contribution of the $s$ quarks to the nucleon charge and magnetization densities.

Part of a worldwide program, the $G^{0}$ experiment is carried out in Hall C of Jefferson Laboratory by a large collaboration of scientists from institutions in the United States, Canada, France, Armenia and Croatia. The ultimate goal of $G^{0}$ is to provide data to allow a comprehensive and precise map of the neutral weak form factors of the proton over the range of momentum transfers $0.1-1.0(\mathrm{GeV} / \mathrm{c})^{2}$. This is done by measuring PV asymmetries in the scattering of polarized electrons on hydrogen and deuterium targets. To allow a separation of the different contributions (electric, magnetic, axial), the $G^{0}$ experimental program is now performing its backward angle measurements. In the coming months, it will provide results for two $Q^{2}$ values, 0.23 and $0.62(\mathrm{GeV} / \mathrm{c})^{2}$. During these measurements, with

a Jefferson Lab Experiments E-05-108/06-108 and E-04-101. A list of collaborators and information about the experiment can be found at http://www.npl.uiuc.edu/exp/G0/

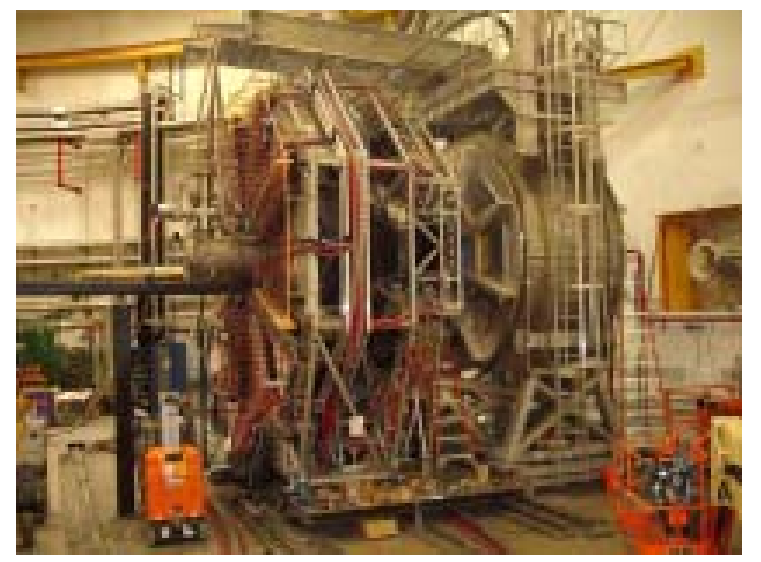

Fig. 1. Photograph of the the G0 set-up installed in Hall C in its "backangle" mode.

a single experimental configuration, data are also recorded for inelastic electron scattering.

\section{Experimental set-up}

A photograph of the overall set-up, after the $180^{\circ}$ rotation of the spectrometer necessary for the backangle running, is shown in figure 1. The heart of the G0 apparatus [6] is a superconducting toroidal magnet (SMS) that focuses scattered particles through collimators onto a focal plane array of scintillators. The magnet contains eight superconducting coils splitting the magnet and detector system into octants. Each octant is defined by collimators placed near the target with an acceptance of $20^{\circ}$ in azimuthal angle. Combined with the polar angle acceptance, the device has a total solid angle of about $0.9 \mathrm{sr}$. The polarized electrons are scattered from a high power cryogenic target (liquid 
hydrogen or deuterium) of $20 \mathrm{~cm}$ length [7]. Currents up to $60 \mu \mathrm{A}$ are used for the backangle measurements with negligible observed target density fluctuations.

The $G^{0}$ octant detection systems are composed of detectors that count either recoil protons from small angle $\left(7-13^{\circ}\right)$ e-p scattering (initial orientation of the magnetic spectrometer), or scattered electrons from large angle $\left(110^{\circ}\right)$ e-N scattering (the backangle phase of $G^{0}$ presented here). A set of 16 detectors in each octant, used in both measurements, lies near the focal surface of the SMS magnet. These detectors (referred to as the Focal Plane Detectors, or FPDs), consist of pairs of plastic scintillators with phototubes at both ends. In the case of the forward angle measurements, particle identification was determined by a time of flight technique using a special $32 \mathrm{~ns}$ beam time structure. This technique is not suitable for backward angle measurements where one has to discriminate between elastic electrons and electrons or pions produced in inelastic processes with similar velocities. The standard CEBAF 2 ns beam time structure was then restored and a second array formed by 9 scintillators was added near the exit window of the magnet cryostat. For each of the eight octants, these detectors (CEDs, for Cryostat Exit Detectors) are used in coincidence with the Focal Plane detectors. This CED/FPD combination correlates the momentum and scattering angle of the detected electrons after its path in the SMS magnetic field, and thus allows for the separation of elastic and inelastic events. With front-end electronics composed of gate arrays, it is possible to record events for given CED/FPD combinations, and thereby measure asymmetries for both elastic and inelastic events. However, simulations and analysis of test data [8] suggested that additional discrimination of electrons and pions was necessary. The $\pi^{-}$background from $n\left(e, \pi^{-}\right) e^{\prime} p$ reactions can indeed be reduced by introducing cuts in CED-FPD space, but not to a level sufficient to isolate quasi-elastic electrons.

Therefore an aerogel Cherenkov counter has been designed to provide pion rejection across the momentum range of the full $G^{0}$ program, up to at least $\sim 400 \mathrm{MeV} / \mathrm{c}$ for $Q^{2}=0.8 \mathrm{GeV}^{2}$. This, of necessity, must be an eightsectored array of individual Cherenkov detectors mounted in conjunction with the CED-FPD sectors. The Cherenkov counters have been located between the CEDs and FPDs. Negatively charged particles entering an octant of the $G^{0}$ spectrometer pass through $5.5 \mathrm{~cm}$ of aerogel (SP30 from Matsushita Electronics). With the chosen index of refraction of the aerogel $(n=1.03)$, pions up to a momentum of $570 \mathrm{MeV} / \mathrm{c}$ do not produce any light whereas all primary electrons do. The photons emitted are collected by four phototubes (XP4572 from Photonis) by means of a diffusion box lined with an internal white reflector. From measurements performed with cosmic muons and test beams at TRIUMF, the electrons were found to generate a signal of 4 to 7 photoelectrons. The detection efficiency obtained in the present data taking is more than $80 \%$ for electrons (when requesting 2 out of the 4 PMTs being fired), whereas a rejection factor of more than 100 to 1 is reached for a $400 \mathrm{MeV} / \mathrm{c}$ pion. The Cherenkov detector

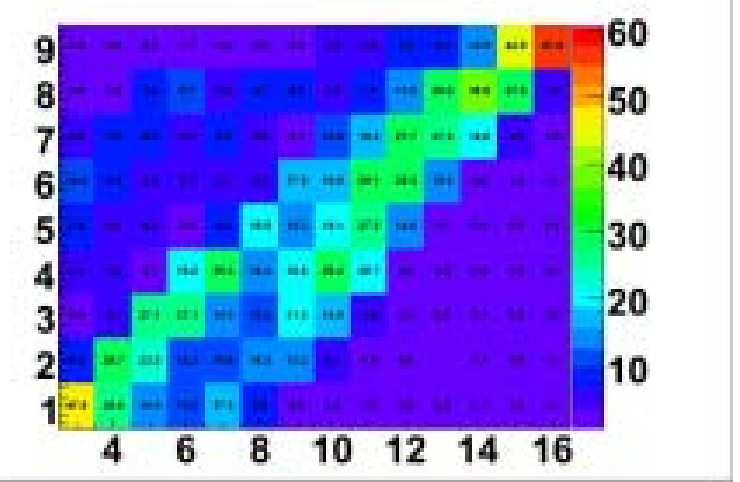

Fig. 2. Counting rates ( $\mathrm{Hz}$ per $\mu \mathrm{A}$ of beam and per octant) in the CED-FPD plane, for particles identified as electrons by the Cherenkov counter, at $\mathrm{Q}^{2}=0.62 \mathrm{GeV}^{2}$

operates in coincidence mode for the electron detection, and in veto mode for background and pion studies.

The $G^{0}$ experiment is also instrumented to measure beam properties (position, charge) in the Hall $\mathrm{C}$ line, and a feedback system at the source level ensures that they are largely independent from the beam helicity state. A new more reliable fiber laser has been used for the present running. It allowed also to switch within a few hours to a $32 \mathrm{~ns}$ beam time structure, used for studies of the Cherenkov performances.

\section{Data taking and first results}

In the backangle phase of the $G^{0}$ experiment, PV asymmetries are measured for several physics cases. The elastic scattering events are detected to access the weak form factors of the nucleon, the inelastic electrons produced in a kinematic range near the $\mathrm{N}-\Delta$ transition [9] to determine the axial mass of the nucleon and finally the pions also produced in this region to study EW corrections [10]. This requires the identification of the three corresponding particles. The experimental set-up for backangle measurements has thus been upgraded with the new detectors discussed above, with corresponding changes in the electronics scheme. The particle identification is then performed by combining information provided by the CEDs, FPDs and Cherenkov detectors. Figure 2 shows an example of the discrimination of the elastic against inelastic electrons in the CED-FPD plane when the Cherenkov is fired (a similar plot is built for pions with the Cherenkov detector being used as a veto).

There have been several experimental difficulties, some only apparent with the first beam in the Hall. First of all, the experiment is running with non-standard, low beam energies (686 and $362 \mathrm{MeV}$ ). This has presented challenges for the accelerator to reach the halo rates and parity quality of the beam required by the measurement. The experiment is using the highly polarized electron beams now produced routinely at Jefferson Laboratory from a supperlattice GaAs cathode. The measurement of the beam po- 

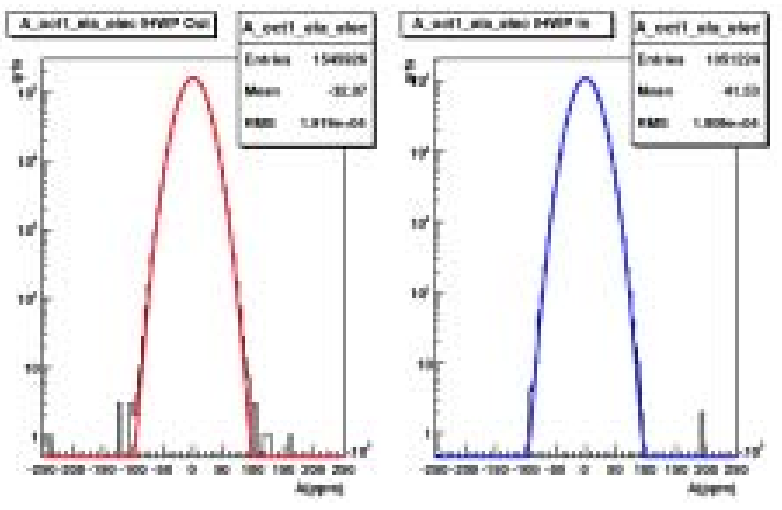

Fig. 3. Distribution of measured detector asymmetries for the two states of the IHWP (see text). A multiplicative blinding factor of $1 \pm 0.25$ has been applied to the data.

larization for these energies required a change in the geometry of the Moeller polarimeter of Hall $\mathrm{C}$ and new operational settings. After Moeller commissioning, the beam polarization (typically $85 \%$ ) was measured for the $686 \mathrm{MeV}$ energy with a precision of 2-3\%, checked during runs made using polarimeters at the injector and in other halls.

A second experimental challenge was related to large singles rates observed in the counters. They are generated mainly by low energy neutrons produced in the target and by moderating processes in the SMS collimators and in the Hall. This has been dealt with for the CED and FPD counters by using additional shielding, achieving better beam conditions and by a change in the FPD phototube arrangement (by placing one PMT from the front scintillator in coincidence with one on the opposite side of the back scintillator). The first data taken have also allowed the tuning of the experimental conditions (magnetic field, thresholds and timing). After these changes, a beam intensity of $60 \mu \mathrm{A}$ is being used for the $\mathrm{LH}_{2}$ target at both energies. The rates obtained are such that losses due to dead time and random coincidences are kept at a reasonable level. It was however not possible to use such beam intensities such beam intensity with a $\mathrm{LD}_{2}$ target for which the production of neutrons is highly enhanced. The limitation comes from the Cherenkov counters for which the very large random coincidence rate observed reduced the effectiveness of the pion rejection. It has been determined that these high rates are due to the capture of thermal neutrons in the glass (Borosilicate) of the Cherenkov phototubes. This has been checked with measurements performed at the NIST facility in Maryland and at the PEREN facility in Grenoble. The solution proposed is to replace the Cherenkov phototubes by new ones with different (quartz) glass windows. From tests with beam in the hall, rates indicate that running with the $\mathrm{LD}_{2}$ target should be possible at the nominal $60 \mu \mathrm{A}$ beam intensity.

There are, at this stage, no physics results to be presented. However the quality of the data being recorded can be estimated from standard PV control spectra like the distribution of the measured detector asymmetries. This is shown in figure 3 for the events selected in the elastic band of the CED-FPD electron plane. As expected, a Gaussian distribution is observed over several orders of magnitude. Also, the sign of the asymmetry is reversed with the insertion of a mechanical device (Insertable Half Wave Plate, IHWP) into the laser path at the source, while, as expected, its amplitude remains unchanged within the error bars.

\section{Conclusions}

After the completion of the forward angle measurements of the $\mathrm{G}^{0}$ program, the experimental set-up was upgraded and installed in its backward angle configuration. The data taking started early 2006 and is presently on schedule until mid-February 2007. Several serious issues have been successfully handled for this measurement. Some of them were related to the use of a non-standard low energy beam (optics and polarimetry) and others to large rates and anode currents due to low energy neutron background. The accelerator has provided beams with the requested parity quality specifications. The running with the liquid Hydrogen target is underway with $60 \mu \mathrm{A}$ beam intensity. The Deuterium target running will follow, once the phototubes in the Cherenkov counters have been replaced by new ones having a quartz window.

With the recording of new backward angle data at two different $\mathrm{Q}^{2}$ values, the physics program of the G0 experiment is nearing completion. The new data will be combined with the data already measured at forward angles, and also with the new measurements foreseen by the HAPPEx and A4 collaborations. The set of data then available should allow one to place strong constraints on the elusive role of the strange sea quarks in the nucleon electromagnetic structure.

Acknowledgments: This work has been made possible by many years of effort from the $G^{0}$ collaborators, and with essential support from the accelerator, target and Hall C groups at Jefferson Laboratory.

\section{References}

1. Proceedings of the PAVI04 Conference, Published in Eur. Phys. J. A., 24 (2005)

2. T. Ito et al., Phys. Rev. Lett. 92, 102003 (2004);

D.T. Spayde et al., Phys. Lett. B 583, 79 (2004).

3. K. Aniol, et al., Phys. Rev. C 69, 065501 (2004); Phys. Lett. B635 275 (2006); Phys. Rev. Lett. 96, 022003 (2006)

4. F. Maas, et al., Phys. Rev. Lett. 93, 022002 (2004); Phys. Lett. B583, 79 (2004) ; Phys. Rev. Lett. 94, 152001 (2005)

5. D. Armstrong et al., Phys. Rev. Lett. 95, 092001 (2005). and the contribution of J.S. Réal in these proceedings.

6. For details see the PhD thesis of J. Liu (University of Maryland, April 2006) and B. Guillon (University of Grenoble, December 2005).

7. S. Covrig et al, Nucl. Instr. Meth. A551, 218 (2005)

8. R. Tieulent et al, G0-Reports G0-01-012 and G0-01-022

9. JLab E-04-101 Experiment, S. Wells and N. Simicevic spokespersons

10. JLab E-04-115 Experiment, J. Martin spokesperson 